\title{
POSITIVE DEFINITE BOUNDED MATRICES AND A CHARACTERIZATION OF AMENABLE GROUPS ${ }^{1}$
}

\author{
MAREK BOŻEJKO
}

ABSTRACT. We show that a discrete group $G$ is amenable iff the Herz-Schur multiplier algebra $B_{2}(G)$ coincides with the Fourier-Stieltjes algebra $B(G)$.

1. Introduction and notation. Let $X$ be a set. A bounded function $a: X \times X \rightarrow \mathbf{C}$ is called positive definite if, for any $\alpha_{i} \in \mathrm{C}$ and any finite $F \subset X$,

$$
\sum_{i, j \in F} \alpha_{i} \bar{\alpha}_{j} a(i, j) \geqslant 0 \text {. }
$$

We denote by $p(X)$ the set of all positive definite bounded functions on $X \times X$.

It was shown by Schur that if $a, b \in p(X)$, then the Hadamard product $a \cdot b \in p(X)((a \cdot b)(i, j) \stackrel{\text { def }}{=} a(i, j) b(i, j), i, j \in X)$.

From that result follows that the set $\mathscr{L}\left(l_{2}(X)\right)$ of all bounded operators on $l_{2}(X)$ forms a Banach algebra under the Hadamard product.

Let $V_{2}(X)$ denote the algebra of all multipliers of the Banach algebra $\mathscr{L}\left(l_{2}(X)\right)$ under the pointwise multiplication, i.e.

$$
V_{2}(X)=\left\{a: a \cdot \mathscr{L}\left(l_{2}(X)\right) \subset \mathscr{L}\left(l_{2}(X)\right)\right\} .
$$

If $S$ and $T$ are two spaces of functions on some set $X$, let $M(S, T)$ denote the space of all multipliers from $S$ into $T$, i.e. the space of the functions $k$ on $X$ such that $k \cdot f \in T$ for every $f \in S$. For $M(S, S)$ we write $M(S)$.

A. Grothendieck [8] observed that

$$
V_{2}(X)=M\left(c_{0}(X) \hat{\otimes} c_{0}(X)\right) .
$$

J. E. Gilbert [6] and G. Bennet [1] showed that $V_{2}(X)=\{\langle x(i), y(j)\rangle: x(i)$, $y(j) \in$ Hilbert space and $\|x(i)\| \leqslant C,\|y(j)\| \leqslant C\}$.

From the last theorem follows that $V_{2}(X)$ is the linear span of the $p(X)$.

The last space was investigated in an excellent way by M. G. Krein [10].

The Littlewood inequality (essentially its dual form) says that if the matrix $a$ defines a continuous linear operator from $l_{1}(X)$ to $l_{2}(X)$, then $a \in V_{2}(X)$, and

$$
\|a\| v_{2} \leqslant \sqrt{2} \sup _{i \in X}\left(\sum_{j \in X}|a(i, j)|^{2}\right)^{1 / 2} .
$$

Received by the editors July 16, 1984.

1980 Mathematics Subject Classification. Primary 20E05, 22B05, 43A07, 43A15.

Key words and phrases. Fourier-Stieltjes algebra, Herz-Schur multipliers, amenable groups, Littlewood functions.

${ }^{1}$ This work was done during a visiting stay at the University of Heidelberg, June-July 1984. I want to thank Professor M. Leinert for his warm hospitality and a nice working atmosphere. 
N. Varopoulos [16] considered the set of Littlewood functions

$$
t_{2}(X)=\left\{a_{1}+a_{2}: a_{1} \in \mathscr{L}\left(l_{1}, l_{2}(X)\right), a_{2} \in \mathscr{L}\left(l_{2}, l_{\infty}(X)\right)\right\}
$$

and he showed that $a \in t_{2}(X)$ iff the norm

$$
\|a\|_{t_{2}}^{2}=\sup _{F_{1}, F_{2} \text {-finite }}\left\{\frac{1}{\left|F_{1}\right|} \sum|a(i, j)|^{2}: i \in F_{1}, j \in F_{2},\left|F_{1}\right|=\left|F_{2}\right|\right\}
$$

is finite.

From now on let $X=G$ be a discrete group and let $G$ act on itself by left translation. We call a function $a$ on $X \times X$ invariant if $a(g x, g y)=a(x, y)$ for any $x, y, g \in G$, i.e. there exists a function $f$ on $G$ such that $f\left(y^{-1} x\right)=a(x, y)$. Let

$$
V N(G)=\mathscr{L}\left(l_{2}(G)\right)^{\text {inv }}
$$

be the set of all invariant operators on $l_{2}(G)$ - the von Neumann algebra of the group $G$ considered by $\mathrm{P}$. Eymard, which is the dual of the Fourier algebra $A(G)=l_{2}(G) * l_{2}(G)$.

Let $B_{2}(G)=V_{2}(G)^{\text {inv }}$ denote the Herz-Schur algebra. For another interesting characterization of the Herz-Schur algebra $B_{2}(G)$ see the paper [3], where it was shown that $B_{2}(G)=M_{0} A(G)$, where $M_{0} A(G)$ is the space of all completely bounded multipliers of the Fourier algebra $A(G)$. For $M_{0} A(G)$ see also the paper [4] of de Cannière and U. Haagerup.

Let $P(G)=p(G)^{\text {inv }}$ be the set of all positive definite functions on $G, B(G)=$ $\operatorname{lin} P(G)$.

Let us note that $B(G)$ is the dual of the full $C^{*}$-algebra $C^{*}(G)$ of the group $G$.

It was proved by $M$. G. Krein [10] that if $G$ is an amenable group, then $B(G)=B_{2}(G)$.

It was shown by C. Herz [9] that, for any locally compact group $G$,

$$
B(G) \subset B_{2}(G) \subset M(A(G)) \text {. }
$$

For the free group $\mathbf{F}_{2}$, M. Leinert [11] has observed that $B\left(\mathbf{F}_{2}\right) \varsubsetneqq B_{2}\left(\mathbf{F}_{2}\right)$; also in the paper [2] it was noted that $B_{2}\left(\mathrm{~F}_{2}\right) \subsetneq M\left(A\left(\mathrm{~F}_{2}\right)\right)$.

C. Nebbia [13] proved that a discrete group $G$ is amenable iff $B(G)=M(A(G))$. V. Losert [18] extended that result to all 1.c. groups.

The aim of this note is to replace Nebbia's result by the following stronger statement:

$$
B(G)=B_{2}(G) \text { iff the group } G \text { is amenable. }
$$

Let us now recall that the Banach space $W$ is called of cotype 2 if there exists a constant $C>0$ such that, for any $x_{1}, x_{2}, \ldots, x_{n} \in W$ and any $n=1,2,3, \ldots$, we have

$$
\int_{0}^{1}\left\|\sum_{k=1}^{n} r_{k}(t) x_{k}\right\| d t \geqslant C\left(\sum_{k=1}^{n}\left\|x_{k}\right\|^{2}\right)^{1 / 2}
$$

where $r_{n}$ are the Rademacher functions on $[0,1]$. It is well known that $L^{p}$-spaces $(1 \leqslant p \leqslant 2)$ are of cotype 2 . 


\section{The Theorem.}

THEOREM. For a discrete group $G$ the following conditions are equivalent:

(i) $G$ is an amenable group.

(ii) $B_{2}(G)=B(G)$.

(iii) $B_{2}(G)$ is of cotype 2 .

Proof. (i) $\rightarrow$ (ii) was given by M. G. Krein [10]; (ii) $\rightarrow$ (iii) follows from the N. Tomczak-Jaegermann [15] theorem that the dual of a $C^{*}$-algebra is of cotype 2. (See also G. Pisier [14] for a simple proof of that fact.) We show now that (iii) $\rightarrow$ (i).

First we show that $M\left(l_{\infty}(G), B_{2}(G)\right) \subset l_{2}(G)$. Let $g \in M\left(l_{\infty}(G), B_{2}(G)\right)$ and let $g=\sum_{n=1}^{\infty} \alpha_{n} \delta_{x_{n}}$, then for each $t \in[0,1]$ the function

$$
g_{t}=\sum_{n=1}^{\infty} \alpha_{n} r_{n}(t) \delta_{x_{n}} \in B_{2}(G) \text { and }\left\|g_{t}\right\|_{B_{2}} \leqslant\|g\|_{M\left(l_{\alpha}, B_{2}\right)} \text {. }
$$

Since by assumption $B_{2}(G)$ has cotype 2 we get

$$
\|g\|_{M\left(l_{\infty}, B_{2}\right)} \geqslant \int_{0}^{1}\left\|\sum \alpha_{n} r_{n}(t) \delta_{x_{n}}\right\|_{B_{2}} d t \geqslant C\left(\sum_{n=1}^{\infty}\left|\alpha_{n}\right|^{2}\right)^{1 / 2} .
$$

Hence $g \in l_{2}(G)$.

Now let us observe that the set of all Littlewood functions

$$
T_{2}(G)=t_{2}^{\mathrm{inv}}(G) \subset M\left(l_{\infty}(G), B_{2}(G)\right),
$$

therefore $T_{2}(G) \subset l_{2}(G)$. On the other hand, we always have $l_{2}(G) \subset T_{2}(G)$, so $T_{2}(G)=l_{2}(G)$.

Let us note (see also J. Wysoczański's general result [17]) that from the Varopoulos characterization of the Littlewood functions we have, for $f \in T_{2}(G)$,

$$
\|f\|_{T^{2}}^{2}=\sup _{\left|F_{1}\right|=\left|F_{2}\right|<\infty}\left\{\frac{1}{\left|F_{1}\right|}\left\langle|f|^{2}, \chi_{F_{1}} * \check{\chi}_{F_{2}}\right\rangle\right\} \leqslant\left\||f|^{2}\right\|_{V N(G)} .
$$

Since $T_{2}(G)=l_{2}(G)$ we get

$$
\||f|\|_{l_{2}(G)}^{2} \leqslant C_{1}^{2}\left\||f|^{2}\right\|_{V N(G)} \quad \text { for every } f \in T_{2}(G) .
$$

This implies that for any positive function $g \in l_{1}(G)$ we have

$$
\|g\|_{l_{1}(G)} \leqslant C\|g\|_{V N(G)} \text {. }
$$

Hence by the Kesten-Hulanicki characterization of amenable groups we obtain that $G$ is amenable.

\section{REFERENCES}

1. G. Bennett, Schur multipliers, Duke Math. J. 44 (1977), 603-639.

2. M. Bożejko, Remark on Herz-Schur multipliers on free groups, Math. Ann. 258 (1981), 11-15.

3. M. Bożejko and Gero Fendler, Herz-Schur multipliers and completely bounded multipliers of the Fourier algebra of a locally compact group, Boll. Un. Mat. Ital. A (6) 3 (1984), 1275-1280.

4. J. de Cannière and U. Haagerup, Multipliers of the Fourier algebras of some simple Lie groups and their discrete subgroups, preprint, 1982.

5. P. Eymard, L'algèbre de Fourier d' un groupe localement compact, Bull. Soc. Math. France 92 (1964), 181-236.

6. J. E. Gilbert, Convolution operators and Banach space tensor products. I, II, III, preprints.

7. F. P. Greenleaf, Invariant means on topological groups, Van Nostrand, New York, 1969. 
8. A. Grothendieck, Résumé de la théorie métrique des produits tensoriels topologiques, Bol. Soc. Mat. Brasil. 8 (1956), 1-79.

9. C. Herz, Une généralization de la notion de transformée de Fourier-Stieltjes, Ann. Inst. Fourier (Grenoble) 24 (1974), 145-157.

10. M. G. Krein, Hermitian-positive kernels on homogeneous spaces. I and II, Ukrain. Mat. Ẑ. 1 (1949), 64-98 and 2 (1950), 10-59; English transl., Amer. Math. Soc. Transl. (2) 34 (1963), 69-164.

11. M. Leinert, Abschätzung von Normen gewisser Matrizen und eine Anwendung, Math. Ann. 240 (1979), 13-19.

12. J. E. Littlewood, On bounded bilinear forms in an infinite number of variables, Quart. J. Math. Oxford Ser. (1) 1 (1930), 164-174.

13. C. Nebbia, Multipliers and asymptotic behaviour of the Fourier algebra of non-amenable groups, Proc. Amer. Math. Soc. 84 (1982), 549-554.

14. G. Pisier, Grothendieck's theorem for non-commutative $C^{*}$-algebras with an appendix on Grothendieck's constant, J. Funct. Anal. 29 (1978), 397-415.

15. N. Tomczak-Jaegermann, On the moduli of smoothness and convexity and the Rademacher averages of the trace classes $S_{p}(1 \leqslant p<\infty)$, Studia Math. 50 (1974), 163-182.

16. N. Th. Varopoulos, On an inequality of von Neumann and an application of the metric theory of tensor products to operator theory, J. Funct. Anal. 16 (1974), 83-100.

17. J. Wysoczański, Characterization of amenable groups and the Littlewood function on free groups, preprint.

18. V. Losert, Properties of the Fourier algebra that are equivalent to amenability, preprint.

Institute of Mathematics, Wroclaw University, Plac Grunwaldzki 2/4, Wroclaw, POLAND 